\title{
RHESSI X-ray loop and coronal sources of an occulted flare
}

\author{
Y. P. Li and W. Q. Gan \\ Purple Mountain Observatory, Nanjing 210008, China \\ email: yplee@pmo.ac.cn
}

\begin{abstract}
An occulted flare occurred at about 06:07 UT on November 2, 2002. The RHESSI X-ray images show two separate parts. The lower part consists of a complete loop and the upper part a coronal source which well extends above the solar limb. The loop source shrank for about 4 minutes with a speed of $\sim 24 \mathrm{~km} \mathrm{~s}^{-1}$ during the early impulsive phase and then expanded at $\sim 7 \mathrm{~km} \mathrm{~s}^{-1}$, while the coronal source presented an upward motion at about $6 \mathrm{~km} \mathrm{~s}^{-1}$. The temperature of the loop-top is always higher than that near the footpoints. The temperature of the loop increased with altitude, while that of the coronal source just showed opposite variation. These results indicate that the reconnection X-point of this flare is located in between the loop-top source and the coronal source.
\end{abstract}

Keywords. Sun: flares, Sun: X-rays, gamma-rays, Sun: corona

\section{Introduction Observations}

The flare region is spatially divided into two parts by reconnection X-point in the classical reconnection model. Careful studies of motions of X-ray sources show that the loop-top source under the X-point moves downward first at the early impulsive phase of a flare and then upward (Sui \& Holman, 2003; Li \& Gan, 2005; Veronig, et al. (2006)). Sui \& Holman (2003) found that the higher energy source in loop source region is at higher altitude whereas that in coronal source region is at lower altitude, and supposed that the temperature variation with source altitude of the coronal source is opposite to that of underlying loops and the current sheet is located between the two sources.

The C3.9 flare occurred from 05:28 UT to 07:04 UT and peaked at 06:07 UT according to GOES records (http://solar.sec.noaa.gov/, SEC). RHESSI and EIT images indicate that it is an occulted flare. We make RHESSI images with the CLEAN imaging algorithm and only using detectors $3 \mathrm{~F}-6 \mathrm{~F}$ and $8 \mathrm{~F}$.

There are two well separated X-ray sources in RHESSI images, We call low-altitude main loop source ' $\mathrm{M}$ ' and the upper coronal source ' $\mathrm{C}$ ', and labeled them in Fig. 1(a). The temperature curves for loop-top and the two regions near the foot of the loop source ' $\mathrm{M}$ ' were obtained by fitting image spectrums in the range of $3-10 \mathrm{keV}$ with $1 \mathrm{keV}$ step. The temperature in loop-top is the highest in the three regions. There is a time delay of the temperature variation between the loop-top and the other part of the loop source.

The centroids of sequential loop-top and coronal sources show that the loop-top moves upward first and then downward in the impulsive phase, the coronal source move upward in the decaying phase.

There are enough photons to reconstruct the loop and coronal temperature map by fitting the image spectrum in the range of $3-10 \mathrm{keV}$ with $1 \mathrm{keV}$ step. The spatial distribution of temperature is presented in Fig. 1(b). For the loop source, the site is nearer the loop-top the temperature is higher. The maximum temperature is about $22 \mathrm{MK}$. For the coronal source the temperature decreases with altitude. 

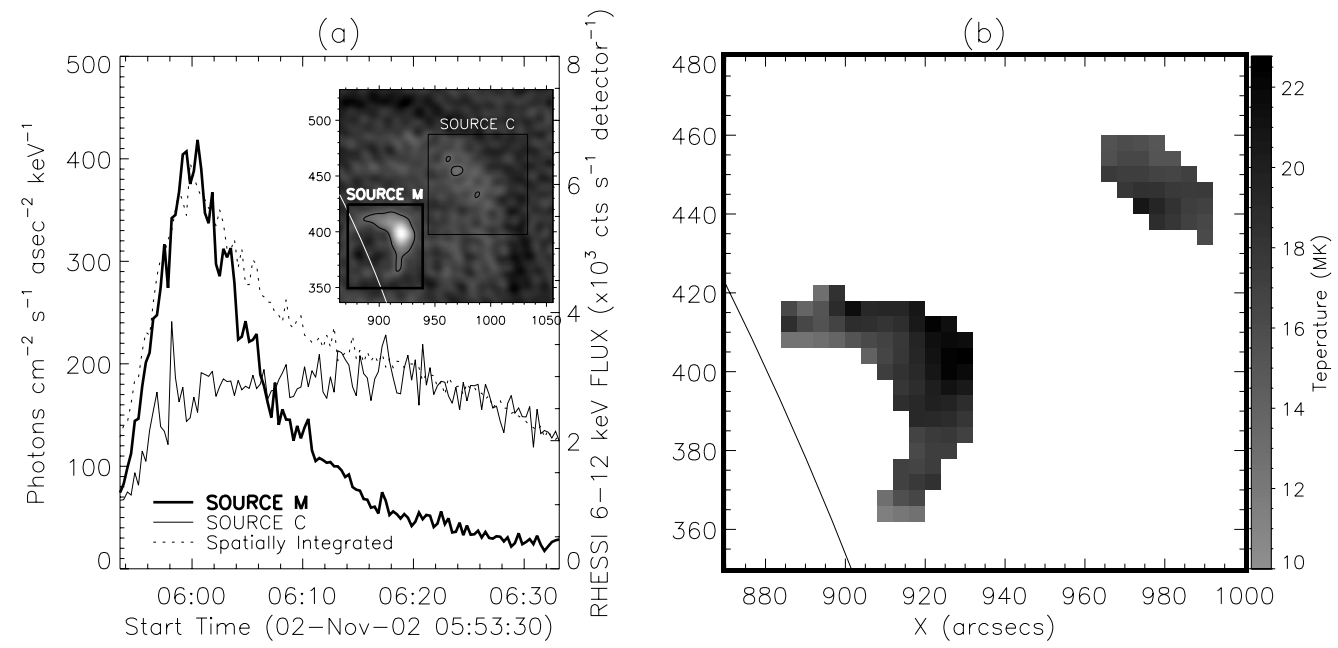

Figure 1. (a) RHESSI 6 - $12 \mathrm{keV}$ light curves for two source regions and the spatially integrated light curve; (b) Image of temperature derived from RHESSI image spectrum in the period of 05:57 - 06:01 UT for the lower source 'M' and 06:16-06:20 UT for the upper source 'C'.

We estimate the conduction cooling time $\tau_{c}$ and the radiation cooling time $\tau_{r}$ for the loop source and coronal one. For the loop source ' $\mathrm{M}$ ', $\tau_{c} \sim 70 \mathrm{~s}, \tau_{r} \sim 6.5 \times 10^{4} \mathrm{~s}$, while for the coronal source ' $\mathrm{C}$ ', $\tau_{c} \sim 2.7 \times 10^{3} \mathrm{~s}$ and $\tau_{r} \sim 3.2 \times 10^{4} \mathrm{~s}$.

\section{Discussions and Conclusions}

The occulted flare of 2002 November 2 consists of two well separated parts: the underlying loop and overlying coronal sources. The light curve of the loop source presents a pulsed shape, while the flux of coronal one changes gradually. The temperature of looptop is higher than that of the lower part of the loop. Furthermore there is a time delay of the temperature variation between the loop-top and the other part of the loop source. The loop-top moves downward quickly in 3 minutes and then upward slowly. The coronal source move upward slowly at all the valid observation times of RHESSI. The plasma temperature at the bottom of the coronal source is higher than that in the upper part. All the results suggest that the X-point of this flare is located between the HXR loop and the coronal sources.

Although this flare is a small GOES event, it's duration is quite long. About two hours after the flare onset, HXR emission was still observable in between the primary loop and coronal sources. The estimation of the cooling time supports that the coronal source can last for a long time.

\section{Acknowledgements}

This work is supported by the National Natural Science Foundation of China via grants 10333040 and 10221001.

\section{References}

Li, Y. P., \& Gan, W. Q. 2005, ApJ (Letters) 629, L137

Sui, L., \& Holman, G. D. 2003, ApJ (Letters) 596, L251

Veronig, A. M., et al. 2006, A\&A 446, 675 